\title{
Mapping refugee litters in the eastern coast of Lesvos using UAS, an emerging marine litter problem
}

\author{
Papakonstantinou, Apostolos ${ }^{\mathrm{a}, *}$, Topouzelis, Konstantinos ${ }^{\mathrm{b}}$, Doukari, Michaela ${ }^{\mathrm{c}}$, Andreadis, \\ Olympos ${ }^{\mathrm{c}}$ \\ ${ }^{a}$ Department of Marine Sciences / Aegean University, apapak@geo.aegean.gr \\ ${ }^{b}$ Department of Marine Sciences / Aegean University, topouzelis@marine.aegean.gr \\ ${ }^{c}$ Department of Marine Sciences / Aegean University, m.doukari@marine.aegean.gr \\ * Corresponding author
}

Keywords: UAS, Marine Litter, Refugee crisis, Orthophoto maps

\begin{abstract}
:
In the last year, the Greek islands of the eastern Aegean Sea were the main entrance points used by refugees arriving in the EU. The crossing between Turkey and the eastern Aegean islands is carried out using mostly small inflatable boats. Additionally, wooden and plastic vessels of various sizes are abandoned at the beaches upon arrival together with other items such as life jackets, inflatable tubes, and clothing. In many cases, these boats are sunk offshore, and their wrecks litter the seabed. This new type of marine litter has overwhelmed many of the eastern Aegean island beaches. In this study, a methodology using Unmanned Aerial Systems (widely known as UAS, UAVs or drones) are used for surveying and mapping of the location and the concentration of marine litter in the coastal areas. More specific is presented the methodology of the mapping of two beaches in Lesvos Island that were surveyed for marine litter, and immigration-related items. One beach is located in the southeaster part and is named Kratigos, and the other one is in the Northeast part of Lesvos close to Korakas cape a widely used "destination" for immigrants due to the lighthouse that is located in this area. The data acquisition has been performed with the use of a Vertical Take-off and Landing (VTOL) quadrotor configuration and the creation of detailed orthophoto maps by processing the aerial photos using Structure from Motion methods and algorithms. The final results of sea marine litter mapping are two very detailed with a high spatial resolution orthophoto maps that are depicting the location, the density and the size of selected litter located underwater and, on the shore, (Figure 1). The main scope of this work was to evaluate: (i) the extent of the refugee arrival related marine litter problem along the eastern coast of Lesvos; and (ii) the efficiency and costeffectiveness of new technologies to provide quick, accurate cartographic visualizations of the marine litter distribution in order to plan efficiently the clean-up operations. The study has shown that the eastern coast of Lesbos faces a very significant environmental problem. Wide stretches of the coastline are overwhelmed by marine litter, such as ship wrecks, and PVC boat remains, lifejackets, discarded clothing and personal items, boat engines and engine fragments. It was also found that litter concentrations varied along the coastline in terms of their distribution over the 'dry" (land) beach and the nearshore seabed. In some areas, there was extensive littering of the dry beach but not of the nearshore seabed, whereas other areas, both with good and very poor (or no) access, showed small quantities of litter on the dry beach but extensive littering on the nearshore seabed. The nearshore seabed offshore of cliff coasts which due to complete lack of access have been not used as entrance points was found to be full of different litter as located from the cartographic representations and observed by dedicated dives.
\end{abstract}




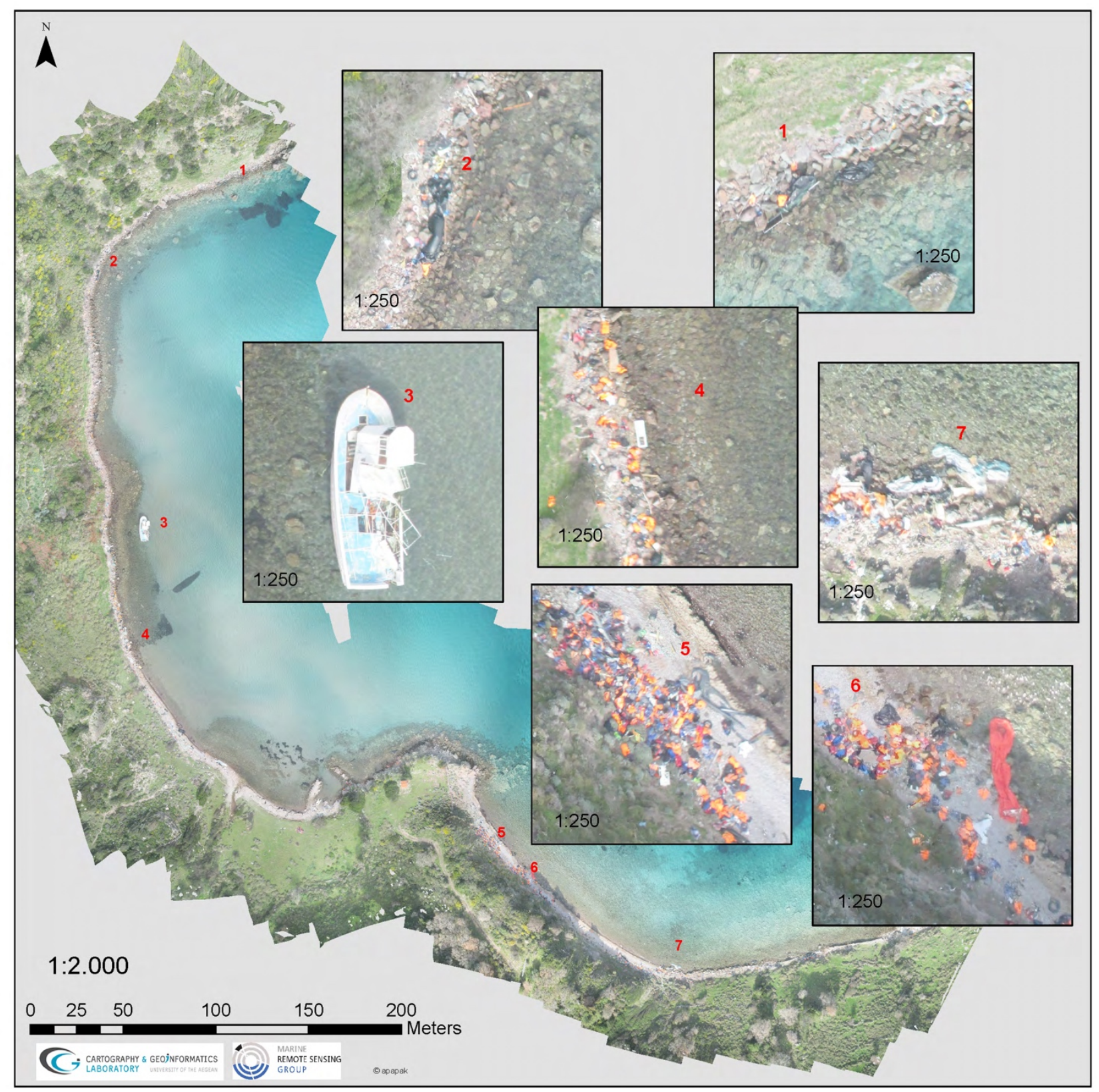

Figure 1. Orthophoto map depicting Tsonia beach and areas with high concentration of refugee litter 